\title{
Effects of Competing Lexical Distracters in a Visual Search Task
}

\author{
Jeremy W. Grabbe, Tom Cioffi, Luke T. Zauner, Kerri L. Driscoll and Sean T. Ford
}

State University of New York, Plattsburgh, New York

Received 2013-11-14; Revised 2014-01-15; Accepted 2014-01-16

\begin{abstract}
Studies have shown that distracters that are relevant to a target can negatively affect visual search performance. Recent studies have looked at performance when targets are embedded within a distracter. Various factors have been explored such as automaticity, spatial location and timing. This study asked, "If distracters affected a different dimension would that produce a detriment to visual search performance"? Would competition for attention occur if distracters were on an irrelevant dimension such as lexicality? The results of this study suggest that lexicality, as demonstrated by word frequency, is a disruptive distracter dimension. This study also showed that two distracters of different word frequencies can serve to disrupt attentional capture.
\end{abstract}

Keywords: Lexical Distracter, Attention Capture, Visual Search

\section{INTRODUCTION}

This experiment expanded on a previous examination of word frequency effects in visual search tasks (Grabbe, 2014) by allowing a simultaneous comparison of distracter words with embedded targets and distracter words without embedded targets within the same trial. This facilitated a greater examination of comparative word frequency effects as distracters (example, the target could be embedded within a low frequency word in a letter string which also contains a high frequency word).

This study will answer the question: "If there was a proximity effect that disrupted the effect of lexicality, would the distracter influence of lexicality still be disruptive when proximity (embeddedness) is also a factor". This will allow a thorough examination of holistic effects of distracter words on the target search task. Grabbe showed an advantage for targets embedded within a word and the reverse word frequency effect. This study will examine the proximity dimension of distracters by utilizing targets embedded within a word and have an additional, nonembedded distracter word.

\section{Hypothesis 1}

A holistic bias will lead to longer reaction time for targets embedded within high frequency words.

\section{Hypothesis 2}

High frequency distracter words will cause a greater distraction than low frequency words. Therefore longer reaction times will be observed for high frequency word distracters.

\section{MATERIALS AND METHODS}

\subsection{Participants}

Nineteen participants were recruited from psychology courses at the State University of New York, Plattsburgh. The age range was from 21 to 51 years of age. All participants were screened for normal or corrected-to-normal visions. Informed consent was obtained from all participants.

\subsection{Procedure}

Participants completed a visual search task in which they searched for a target letter within a letter string. The target letter was presented before each trial. In the experiment all targets were embedded within a word or nonword in a letter string. Each letter string also had a second word which served as a distracter and did not have an embedded target.

Corresponding Author: Jeremy W. Grabbe, State University of New York, Plattsburgh, New York 


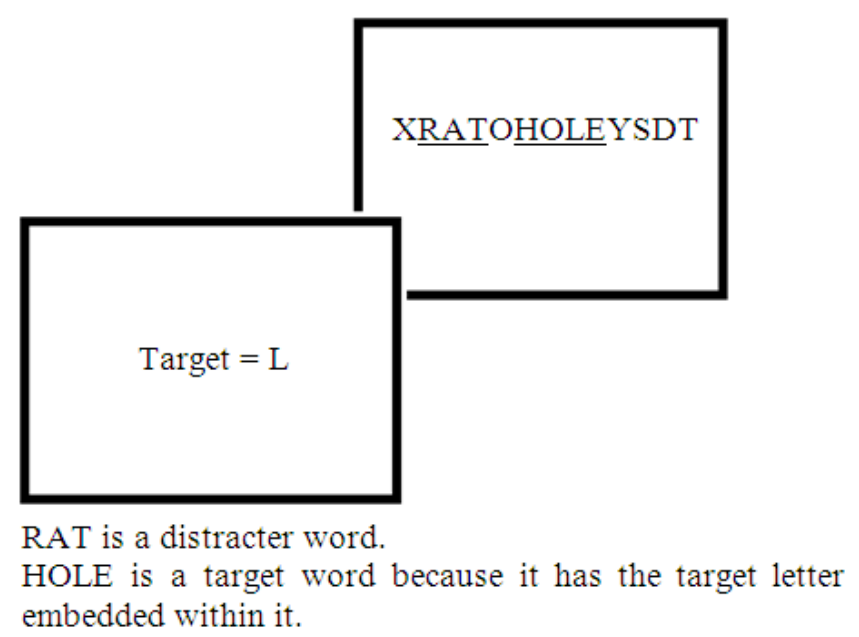

Fig. 1. Stimuli display for the experiment. Underline represents word and italics represents target within the letter string. Neither target nor word were italicized or underlined during the experiment

For the purpose of clarity the distracter in which a target is embedded (which can be a word or nonword) will be referred to as the first distracter and the distracter that does not contain an embedded target (which is always a word) is referred to as the second distracter. Example, the target is the letter " $\mathrm{L}$ " and the letter string is XRATOHOLEYSDT. In that letter string the target appears is embedded in the first distracter (the word "hole") while the word "rat" serves as the second distracter (Fig. 1).

Participants completed 10 practice trials before completing 600 experiment trials. Target present trials were composed of three word frequency categories. Each word frequency category for first distracters contained 160 trials of nonreplaced words along with second distracters that were spread equally across word frequency category. Words from word frequency database of Brysbaert and New (2009) were used in this experiment in the target absent condition, no letter string contained a legal, English word. All trials were randomized. All letter strings remained on the screen until participants pressed the left or right arrow key to indicate if the target letter was present

\section{RESULTS}

\subsection{Accuracy}

Data for a words only analysis (first distracter nonwords were not analyzed) were analyzed in a $3 \times 3$ ANOVA consisting of word frequency of the first distracter (low, medium and high word frequency) and word frequency of the second distracter (low, medium and high word frequency). Nonword and words were compared in a 2 (target: Word Vs. nonword) $\times 3$ (distracter: (low, medium and high word frequency)) ANOVA.

\subsubsection{Words only}

No significant effect for word frequency.

\subsubsection{Nonwords Vs. Words}

No significant effect for word frequency

\subsection{Reaction Time}

\subsubsection{Words Only}

There were no significant main effects for first distracter word frequency or second distracter word frequency. The interaction between first distracter and second distracter word frequency was significant, $\mathrm{F}(4$, $72)=18.43, p<0.01, \eta p 2=0.535$. This was because low frequency first distracters had quicker reaction times when the second distracter was a low frequency word. High frequency first distracter words also showed this effect, but medium frequency first distracter words showed the opposite effect (Fig. 2).

\subsubsection{Words Vs. Nonwords}

There was a significant word/nonword first distracter main effect, $F(1,17)=148.3, p<0.05, \eta p 2=0.897$. Mean first distracter nonword reaction time $(908 \mathrm{~ms})$ was faster than first distracter word reaction time $(932 \mathrm{~ms})$. There was a significant main effect for second distracter word frequency, $\mathrm{F}(2,34)=10.01, \mathrm{p}<0.05$, $\eta \mathrm{p} 2=0.069$ (Fig. 3). 


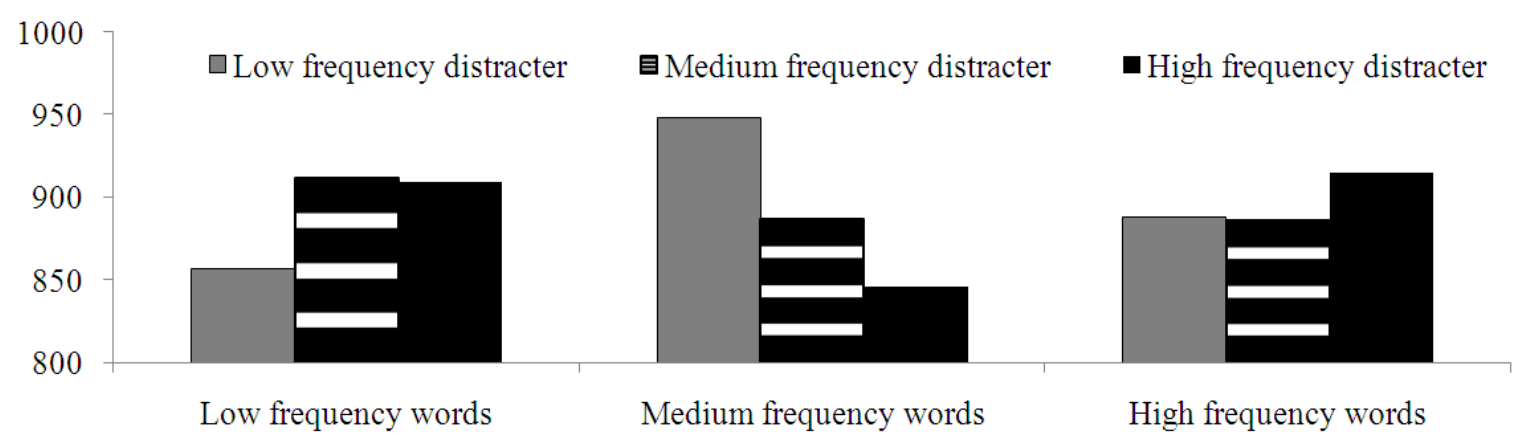

Fig. 2. Mean reaction time by word frequency for letter strings with targets embedded within a word and a word distracter

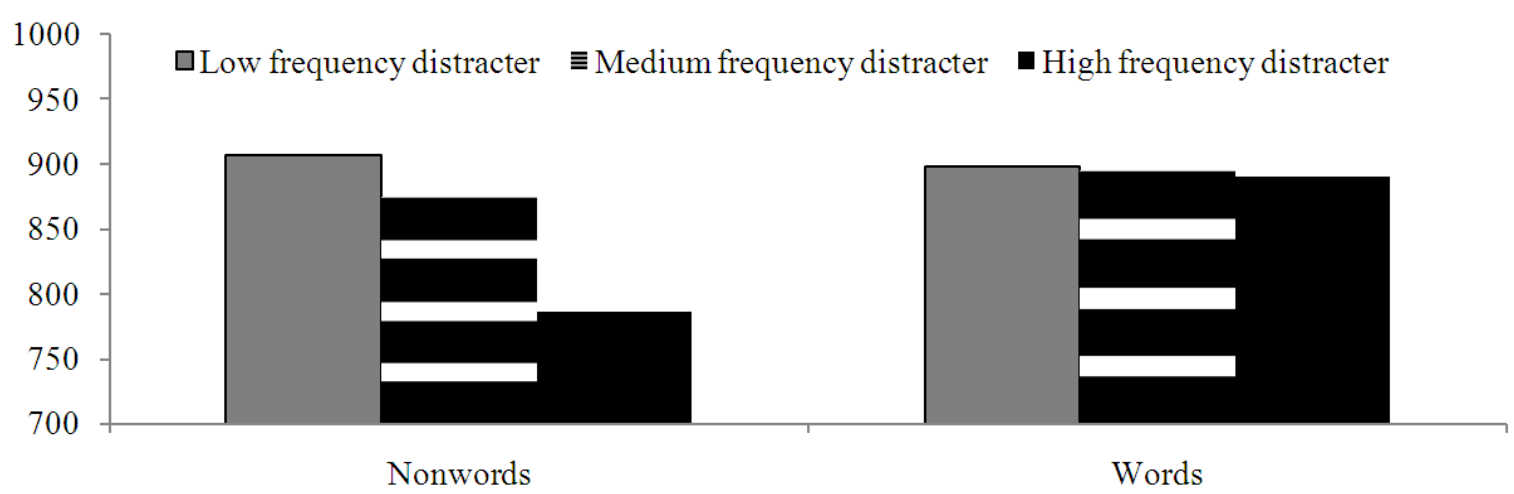

Fig. 3. Mean reaction time by distracter word frequency for letter strings with targets embedded within a word and targets embedded within a nonword

Typically, reaction time decreased as word frequency increased. There was a significant word/nonword first distracter $\mathrm{X}$ second distracter frequency interaction, $\mathrm{F}(2,34)=4.16, \mathrm{p}<0.05, \eta \mathrm{p} 2=0.097$. Although both words and nonwords first distracters showed a reaction time benefit as second distracter word frequency increased this effect was greater for nonword first distracters than word first distracters.

\section{DISCUSSION}

When targets were embedded within a high frequency or low frequency word (first distracters) there were significantly longer reaction times for higher frequency word second distracters where were congruent with Hypothesis 2. Strangely, this effect was reversed for targets embedded within medium frequency words proposing a unique attentional selection process when rejecting distracters. That effect could possibly be the result of a shifting between letter-level processing at low frequency words and holistic processing at high frequency words. Letter strings are categorically distinguished between words and nonwords before processing salience is determined (Ariga and Watanabe, 2009), then endogenous bias towards holistic processing (of words) focuses attention, facilitating letter-level processing. This distracted attention away from low and high frequency words containing a target; thus, longer RT's were recorded for targets embedded within low and high frequency words.

Previous research on attention has demonstrated that distracters have significant costs to performance (Flowers and Lohr, 1985). However, there has been conflicting evidence as to what conditions and distracter characteristics produce larger performance deficits. Chen and Cave (2006) found that a distracter will always be processed unless both the target and distracter locations are already known. Their results would imply top-down inhibition of distracter influence. This negation of distracter effects is in contrast to Neo and Chua (2006). $\mathrm{Neo}$ and Chua found that even when a target location is know the sudden onset of a distracter will capture attention. This phenomenon of automatic distracter processing (Flowers and Smith, 1998; Eriksen and 
Eriksen, 1974) is supported by the results of this study which found automatic processing of distracters affected performance, even when the dimension of the distracter was irrelevant to the task.

A decreased RT in identifying targets when embedded within medium frequency words among high frequency word distracters could suggest an integration of letter-level and holistic processing, or bottom-up and top-down processing working in conjunction as slightly explored in previous literature (Hickey and Theeuwes, 2011). Inukai et al. (2010) also discovered a similar effect by manipulating the duration of peripheral distracters while identifying a central visual target. Their results suggest top-down and bottom-up processes simultaneously affected (Inukai et al., 2010) the feature search mode during target identification. In the present study, this could contradict a holistic processing bias towards high frequency words among medium frequency words. These results also offer the conclusion that the benefit of targets embedded in word distracters is reduced by additional, non-embedded word distracters and that lexicality is a stronger influence in distracter effects than proximity. However, such a debate is beyond the scope of this study and would be an area best pursued by future research.

\section{CONCLUSION}

One limitation to this study is that although there is strong evidence of automatic lexical processing the thoroughness of automatic lexical processing cannot be determined from this study. The thoroughness of lexical processing could be a significant factor in the strength of attentional capture. Future research on attentional capture such as this should focus on evaluating thoroughness of lexical processing by utilizing a priming task such as a word stem completion task. This study demonstrated that in a letter search task embedded and nonembedded distracter can affect performance by varied word frequency. This favors automatic lexical processing as a new distracter dimension that may serve as a hindrance or aid to performance depending upon the word frequency (Grabbe, 2014).

\section{REFERENCES}

Ariga, A. and K. Watanabe, 2009. Temporal dissociation between category-based and item-based processes in rejecting distractors. Psychol. Res., 73: 54-59. DOI: 10.1007/s00426-008-0143-9

Brysbaert, M. and B. New, 2009. Moving beyond Kucera and Francis: A critical evaluation of current word frequency norms and the introduction of a new and improved word frequency measure for Am. English Behav. Res. Meth., 41: 997-990. DOI: 10.3758/BRM.41.4.977

Chen, Z. and K.R. Cave, 2006. When does visual attention select all features of a distractor? J. Exp. Psychol. Hum. Percept. Perform., 32: 1452-1464. PMID: 17154784

Eriksen, B.A. and C.W. Eriksen, 1974. Effects of noise letters upon the identification of a target letter in a nonsearch task. Percept. Psychophys., 16: 143-149. DOI: $10.3758 /$ BF03203267

Flowers, J.H. and D.J. Lohr, 1985. How does familiarity affect visual search for letter strings? Percept. Psychophys., 37: 557-567. DOI: 10.3758/BF03204922

Flowers, J.H. and K.L. Smith, 1998. What is learned about nontarget items in simple visual search? Percept. Psychophys., 60: 969-704. DOI: 10.3758/BF03206056

Grabbe, 2014. Effects of targets embedded within words in a visual search task. Adv. Cognitive Psychol.

Hickey, C. and J. Theeuwes, 2011. Context and competition in the capture of visual attention. Attent. Percept. Psychophys., 73: 2053-2064. DOI: 10.3758/s13414-011-0168-9

Inukai, T., T. Kumada and J.I. Kawahara, 2010. Attentional capture decreases when distractors remain visible during rapid serial visual presentations. Attent. Percept. Psychophys., 72: 939950. DOI: 10.3758/APP.72.4.939

Neo, C. and F.K. Chua, 2006. Capturing focused attention. Percept. Psychophys., 68: 1286-1295. DOI: $10.3758 / \mathrm{BF} 03193728$ 